\title{
On the Cosmic Rotation Axis
}

\author{
Rainer W. Kühne \\ Fachbereich Physik, Universität Wuppertal, 42097 Wuppertal, Germany, kuehne@theorie.physik.uni-wuppertal.de
}

\begin{abstract}
Recently, Nodland and Ralston reported to have discovered a cosmic axis. We argue that their axis is supported by an earlier independent observation on the spin axes of galaxies in the PerseusPisces supercluster and explainable within the framework of Gödel's cosmology.
\end{abstract}

PACS number: 98.80.Es

Nodland and Ralston [1] claim to have found evidence that polarized radiation emitted by distant radio galaxies shows residual rotation, remaining after Faraday rotation is extracted, that follows a dipole rule. The residual angle $\beta$ appears to be correlated with the distance $r$ of the radio galaxy and the angle between the propagation wave vector $\mathbf{k}$ of the radiation and the axis $\mathbf{s}$. In sum, the claimed relation for the mean residual angle is

$$
<\beta>=\frac{r}{2 \Lambda_{s}} \cos (\mathbf{k}, \mathbf{s}) .
$$

Expressed in the coordinates $R . A .=$ right ascension and decl. $=$ declination the axis points in the direction

$$
\mathbf{s}=(\text { R.A., decl. })=\left(315^{\circ} \pm 30^{\circ}, 0^{\circ} \pm 20^{\circ}\right) .
$$

Under the assumption of the Einstein-de Sitter cosmology (i. e. critical mean mass density and vanishing cosmological constant) and by setting the present value of the Hubble parameter to

$$
H_{0}=\left(1.5 \times 10^{10} \mathrm{yr}\right)^{-1}=66.7 \mathrm{~km} /(\mathrm{s} \mathrm{Mpc}),
$$

the birefringence scale was found to be

$$
\Lambda_{s}=(1.1 \pm 0.08) \times 10^{25} \mathrm{~m} .
$$

The corresponding universal angular velocity is

$$
\omega=\frac{c}{2 \Lambda_{s}}=1.4 \times 10^{-17} \mathrm{~s}^{-1} .
$$

Such an intrinsic spin would have dramatic consequences, because it would violate Mach's principle which states that, "A system on which no forces act is either at rest or in uniform motion relative to the fixed stars idealized as a rigid system".

A cosmology with intrinsic universal rotation was already suggested by Gödel [2]. Although its original version considers the static universe only, this model becomes viable if universal expansion is included, as shown by Raychaudhuri [3].

Gödel predicted that the original order of the rotation axes of galaxies has been parallel to the universal rotation axis. This idea was supported by the discovery [- 4 that the distribution of the rotation axes for both the spiral and ellipsoid galaxies of the filament-like Perseus-Pisces supercluster is bimodal. One of the peaks is roughly aligned with the major axis of the supercluster while the second peak is roughly $90^{\circ}$ from the first. Quite remarkably, the major axis of this supercluster points in the direction

$$
\mathbf{s}_{p p}=(\text { R.A., decl. })=\left(290^{\circ} \pm 20^{\circ},-20^{\circ} \pm 10^{\circ}\right) .
$$

Within the error bars this axis agrees with the one detected by Nodland and Ralston.

[1] B. Nodland and J. P. Ralston, Phys. Rev. Lett. 78, 3043 (1997).

[2] K. Gödel, Rev. Mod. Phys. 21, 447 (1949).

[3] A. Raychaudhuri, Phys. Rev. 98, 1123 (1955).

[4] S. A. Gregory, L. A. Thompson, and W. G. Tifft, Astrophys. J. 243, 411 (1981). 